\title{
Narrative review of immune checkpoint inhibitors and radiation therapy for brain metastases
}

\author{
Marina Moskalenko, Tyler P. Robin \\ Department of Radiation Oncology, University of Colorado School of Medicine, Aurora, CO, USA \\ Contributions: (I) Conception and design: All authors; (II) Administrative support: All authors; (III) Provision of study materials or patients: All \\ authors; (IV) Collection and assembly of data: All authors; (V) Data analysis and interpretation: All authors; (VI) Manuscript writing: All authors; (VII) \\ Final approval of manuscript: All authors. \\ Correspondence to: Tyler P. Robin, MD, PhD. Assistant Professor, Radiation Oncology, University of Colorado School of Medicine, 1665 Aurora Ct, \\ Suite 1032, MS F706, Aurora, CO 80045, USA. Email: tyler.robin@cuanschutz.edu.
}

\begin{abstract}
Radiation therapy plays a key role in the management of intracranial metastatic disease. Historically, systemic therapy was able to address extracranial disease but not cross the blood-brain barrier and radiation therapy and surgery were the only mechanisms to treat intracranial metastases. There are now several examples of contemporary systemic therapies with central nervous system efficacy in some patients. With such improvements in systemic therapies, patients are living longer and the optimal management of brain metastases is becoming an increasingly important clinical priority. However, the role of radiation therapy remains critical in treating brain metastases. The concurrent use of new systemic therapies with radiation brings about novel and significant questions regarding potential synergy between these therapies in the brain in regard to both oncologic efficacy and toxicity. One important systemic therapy to consider is immune checkpoint inhibitors. These drugs are now at the forefront of management of many malignancies and have changed the landscape of treatment for many common cancers, particularly those with a predilection for brain metastases. In this review we will examine the existing data on the efficacy and toxicity of concurrent radiation therapy and immunotherapy for brain metastases and explore potential mechanisms underlying the published clinical observations.
\end{abstract}

Keywords: Immunotherapy; immune checkpoint inhibitors (ICIs); stereotactic radiosurgery (SRS); brain metastases

Submitted Oct 12, 2020. Accepted for publication Apr 01, 2021.

doi: $10.21037 /$ tcr-20-3027

View this article at: http://dx.doi.org/10.21037/tcr-20-3027

\section{Introduction}

Brain metastases are a challenging complication associated with many advanced solid tumors, and although the magnitude of the problem is difficult to accurately quantify, by most estimates brain metastases far outnumber primary gliomas $(1,2)$. Importantly the prognosis for patients with brain metastases has improved with the most contemporary analyses demonstrating median survival of 33 months [compared with 16.73 months in an older dataset (3)] among patients with brain metastases from breast, non-small cell lung cancer (NSCLC), renal cell carcinoma (RCC), melanoma, and gastrointestinal (GI) cancers, with the best prognostic features (4). With improvements in systemic therapies patients are living longer and the optimal management of brain metastases is becoming an increasingly important clinical priority.

One important systemic therapy to consider is immune checkpoint inhibitors (ICIs). ICIs have dramatically changed the landscape of systemic therapy for many common cancers including those with a high propensity for brain metastases, including melanoma, NSCLC, and RCC (5-7). ICIs appear to have central nervous system (CNS) activity to varying extent yet radiation therapy 
remains a key component of the management of brain metastases and therefore it is critical to understand the implications of concurrent administration of brain radiation and ICIs (8-17). While data on this topic primarily consists of small retrospective studies, in this article we will attempt to consolidate and review the available literature to better understand the potential synergy between these therapies in both toxicity and efficacy and discuss possible mechanisms to explain the findings that have been reported to date. We present the following article in accordance with the Narrative Review reporting checklist (available at http://dx.doi. org/10.21037/tcr-20-3027).

\section{CNS efficacy of ICls alone}

ICIs function to enhance anti-tumor responses by the immune system and have changed the treatment paradigm for several metastatic cancers. The groundbreaking studies that led to many of the approvals for ICIs largely excluded patients with untreated brain metastases (18-29). However, several subsequent studies addressed the role of ICIs in patients with brain metastases and are detailed below.

Margolin et al. conducted a multi-institutional, open-label, phase II study with two parallel cohorts: asymptomatic (cohort A) or symptomatic (cohort B) melanoma brain metastases treated with ipilimumab. CNS disease control rate at 12 weeks was 24 vs. $10 \%$ in cohorts $\mathrm{A}$ and $\mathrm{B}$, respectively. The median overall survival (OS) was 7 months for asymptomatic brain metastases and 3.7 months for symptomatic brain metastases (14). In a separate multi-institutional, open-label, phase II Italian trial, NIBIT-M1, Di Giacomo et al. investigated the efficacy of ipilimumab plus fotemustine in patients with metastatic melanoma with or without asymptomatic brain metastases. Ten of the 20 patients with brain metastases had immune-related disease control. Of note, 7 patients (35\%) had previous radiation with either whole brain radiation therapy (WBRT) or stereotactic radiosurgery (SRS) for brain metastases (16). A follow-up study to assess longterm outcomes showed median survival and 3 -year OS rates were 12.9 months and $28.5 \%$ for the whole study population, and 12.7 months and $27.8 \%$ for patients with brain metastases, respectively (17). NIBIT-M2 is an ongoing (ClinicalTrials.gov: NCT02460068) phase 3, open-label study that is randomizing, metastatic melanoma patients with untreated, asymptomatic brain metastases to fotemustine alone, fotemustine and ipilimumab, or ipilimumab and nivolumab.

The efficacy of pembrolizumab in melanoma brain metastases has also been evaluated. In a two-cohort phase II study, Kluger et al. enrolled 23 patients with untreated asymptomatic melanoma brain metastases (12). Five patients (22\%) had no prior CNS therapy, the rest had either surgical resection, WBRT, or SRS. The brain metastasis response rate was $26 \%$ with 2 partial responses and 4 complete responses.

While single agent ICIs show some efficacy in patients with brain metastases, the intracranial response rates remain variable with control rates still lower than desired and most studies reporting the use of potentially confounding additional local control with either radiation or surgery. A couple of studies have investigated the role of dual agent ICIs in patients with brain metastases with evidence for slightly higher response rates. In an open-label, multicenter, phase 2 study by Tawbi et al., patients with metastatic melanoma and at least one measurable, nonirradiated brain metastasis (tumor diameter, 0.5 to $3 \mathrm{~cm}$ ) and no neurologic symptoms received nivolumab plus ipilimumab (15). Among 94 patients with a median follow-up of 14.0 months, the rate of intracranial clinical benefit, defined as stable disease for at least 6 months, complete response, or partial response, was $57 \%$, with $26 \%$ exhibiting a complete response. Similarly, in a multicenter open-label randomized phase 2 trial Long et al. evaluated the efficacy of the same combination of ICIs compared to nivolumab alone (13). Patients with asymptomatic melanoma brain metastases with no previous local brain therapy were randomly assigned to combination nivolumab plus ipilimumab (cohort A) or nivolumab alone (cohort B); patients with brain metastases in whom local therapy had failed, or who had neurological symptoms, or leptomeningeal disease were non-randomized (cohort C). At a median follow-up of 17 months, observed intracranial responses were $46 \%, 20 \%$, and $6 \%$, for cohorts $\mathrm{A}, \mathrm{B}$, and C, respectively.

Similar to the paradigm changing clinical trials in melanoma, the clinical studies leading to the approval of ICIs in advanced NSCLC also commonly excluded patients with brain metastases (18-20,23,24,29). However, in the two-cohort phase two study referenced above (12), patients with stage IV NSCLC with at least one asymptomatic brain metastasis $5-20 \mathrm{~mm}$ in size, not previously treated or progressing after previous radiotherapy and not requiring corticosteroids were also evaluated $(10,11)$. NSCLC patients were divided into two cohorts: those with PD-L1 expression of at least $1 \%$ (cohort 1 ) or those with PD-L1 less than $1 \%$ or 
unevaluable (cohort 2). At a median follow up of 8.3 months, 11 of 37 patients $(29.7 \%)$ had a brain metastasis response in cohort 1 , but there were no responses in cohort 2 . In a subgroup analysis of the phase III, open-label OAK study investigating the efficacy of atezolizumab versus docetaxel in previously treated patients with NSCLC, Gadgeel et al. focused specifically on patients with asymptomatic, treated supratentorial brain metastasis (30). Median survival was longer with atezolizumab than with docetaxel regardless of brain metastasis status, and patients with a history of asymptomatic, treated brain metastases had a lower probability of developing new symptomatic brain lesions with atezolizumab $v s$. docetaxel (30).

In RCC, 73 patients from the prospective phase II trial, GETUG-AFU 26 NIVOREN, had brain metastases and were included in an analysis of the efficacy of nivolumab in patients with RCC disseminated to the brain who experienced disease progression after VEGFR-directed therapies (9). Patients with asymptomatic brain metastases were prospectively identified and followed in two cohorts. Cohorts A and B included patients with untreated and treated brain metastases, respectively. In cohort A, the intracranial response rate was $12 \%$, although no responses were observed in patients with intracranial tumor burden greater than $1 \mathrm{~cm}$. Most patients in cohort A (72\%) required subsequent focal brain therapy.

Several ongoing prospective clinical trials are currently investigating the efficacy and safety of ICIs as dual agents or in combination with other systemic therapies in patients with brain metastases of various histologies in hopes of expanding on the above data. However, the most effective means of optimizing intracranial benefit might be in combining ICIs with radiotherapy.

\section{Role of radiation in treatment of brain metastasis}

While WBRT was the historical standard for CNS radiotherapy, multiple randomized trials have since demonstrated that while WBRT improves CNS control there is no OS benefit and WBRT is associated with neurocognitive decline. These studies have established SRS as the preferred option for most patients at least with limited brain metastases (8,31-35). Importantly, Yamamoto et al. conducted a single-arm multi-institutional prospective study of SRS alone in over 1,000 patients with 1-10 brain metastases and stratified patients into $1,2-4$, and 5-10 brain metastases. While the longest OS was observed in patients with one brain metastases, there were no significant differences in OS between patients with 2-4 and 5-10 brain metastases, supporting an SRS alone paradigm for patients with multiple brain metastases as well (36). In the following sections covering the combination of radiation therapy and ICIs, we will primarily focus on patients treated with SRS.

\section{ICls and radiosurgery: efficacy and sequencing}

Preclinical data suggests radiation can stimulate immune mediated anti-tumor effects. Tumor irradiation has been shown to enhance neoantigen release, promote proinflammatory signals, dendritic cell recruitment and antigen cross-presentation, and prime tumor specific $\mathrm{T}$ cell responses and increase $\mathrm{T}$ cell tumor infiltration (37-39). However, tumors are capable of evading the host immune system via several mechanisms including checkpoint molecules. The combination of ICIs with SRS has the potential to subvert such immune evasion and enhance treatment response (37-39). Furthermore, radiation therapy can disrupt the blood-brain barrier (BBB) and SRS specifically appears to be a potent means for increasing $\mathrm{BBB}$ permeability (40-42).

Although primarily small retrospective studies, there are an increasing number of reports suggesting an association between receipt of SRS with ICIs and improved intracranial response rate and/or extended survival (43-66). The majority of reports are in patients with melanoma brain metastases with smaller numbers of patients included with NSCLC, RCC, and other primary tumor types. Several early retrospective studies of SRS with or without concurrent ipilimumab actually failed to demonstrate a significant improvement in CNS disease control outcomes $(50,56,57)$. Although for the most part an OS benefit was observed $(50,56,65,66)$.

However, in a multi-institutional retrospective study of ninety-nine melanoma patients that received ipilimumab and then developed new brain metastases and underwent SRS, those that were treated with SRS within 5.5 months after ipilimumab demonstrated improved intracranial disease control. No difference in OS was observed in this study (45). In a study by Ahmed et al., 96 patients underwent SRS for melanoma brain metastases within 3 months of systemic therapy. Systemic therapies included anti-PD-1, anti-CTLA-4, BRAF/MEK inhibitors (BRAF/MEKi), and conventional chemotherapy. One-year distant brain 
metastasis control was highest with concurrent anti-PD-1 (38\%), followed by anti-CTLA-4 (21\%), BRAF/MEKi (20\%), BRAFi (8\%), and conventional chemotherapy (5\%) $(\mathrm{P}=0.008)$ (44). Similarly, Acharya et al. looked at SRS within 3 months of systemic therapy also including ICIs (anti-PD-1 and/or anti-CTLA-4) and targeted therapy (BRAFi with or without MEKi), and found 1-year distant brain metastasis control of $60 \%, 10 \%$, and $11.5 \%$ for SRS and ICIs, SRS and targeted therapy, and SRS alone, respectively. Undergoing SRS with concurrent ICIs was also associated with a decreased likelihood of local failure compared with SRS alone [hazard ratio (HR) 0.37; 95\% CI: 0.14-0.95; $\mathrm{P}=0.04]$ (43). Qian et al. conducted a single institution retrospective study of 75 patients with melanoma brain metastases that received SRS and immunotherapy (anti-CTLA-4: 72\%; anti-PD-1: 28\%). Concurrent (defined as SRS within 4 weeks of immunotherapy) treatment and anti-PD-1 (compared with anti-CTLA-4) both resulted in improved median percent reduction in lesion size (58). Minniti et al. reviewed the outcomes for eighty patients with melanoma brain metastases that received ipilimumab or nivolumab within 1 week of SRS. Patients receiving concurrent nivolumab (compared with ipilimumab) demonstrated improved 12-month intracranial progressionfree survival ( $42 \%$ vs. $17 \%$ ) and OS (78\% vs. $68 \%$ ) (55). Our group retrospectively identified 38 patients that initiated ICI therapy within 8 weeks before or after undergoing SRS for melanoma brain metastases and observed significantly longer time to out-of-field CNS progression in patients that received anti-PD-1 with/without anti-CTLA-4 therapies compared with anti-CTLA-4 alone (median not reached $v s$. 3.1 months) (59).

In NSCLC, a few groups have reviewed the oncologic outcomes of patients receiving ICIs and SRS (60-62). Singh et al. retrospectively reviewed the outcomes for 85 NSCLC patients treated with SRS and immunotherapy versus chemotherapy and found no difference in local tumor response (with the exception of the subset of patients with lesions with volume greater than $500 \mathrm{~mm} 3$ ) or survival (62). Shepard et al. conducted a similar study that included 17 patients (with 45 brain metastases) that received concurrent (within 3 months) SRS and ICIs, compared with 34 patients (92 brain metastases) that received SRS alone. They did report increased rates of lesion complete response and shorter time to brain metastasis regression with concurrent therapy, but no differences in local control, CNS progression-free or OS were observed (61). However, Schapira et al. retrospectively identified 37 NSCLC patients with 85 brain metastases treated at a single institution with SRS and PD-1 pathway inhibitors and observed longer OS and reduced rates of distant brain failure with concurrent (within 4 weeks) ICIs and SRS compared to SRS delivered greater than 1 month before or greater than 1 month after ICIs (1-year OS, $87.3 \%$ vs. $70.0 \%$ vs. $0 \%, \mathrm{P}=0.008$; 1 -year distant brain failure, $38.5 \%$ vs. $65.8 \%$ vs. $100 \%, \mathrm{P}=0.042)(60)$. Although not encompassed in the above studies, ALK and EGFR mutations remain important targets for patients with NSCLC and have shown to be associated with improved survival with targeted agents (4). It is unclear how ICI will play a role for this subgroup of patients with a targeted therapy option.

Chen $\mathrm{et} \mathrm{al}$. evaluated the outcomes of 260 patients with brain metastases from melanoma, NSCLC, and RCC, that received SRS alone, SRS with concurrent ICIs (antiCTLA-4, anti-PD-1, or both; given within 2 weeks of SRS), and SRS with non-concurrent ICIs, and found a decreased likelihood of the development of three or more new brain metastases and improved OS in the concurrent group (47). Lehrer $e t$ al. conducted a meta-analysis of individual patient data that included 534 patients with 1,570 brain metastases (primarily melanoma but also including NSCLC and RCC patients) treated with SRS and ICIs, either concurrently or non-concurrently. Concurrent therapy was associated with improved 1-year OS (64.6\% vs. 51.6\%, $\mathrm{P}<0.001), 1$-year local control $(89.2 \%$ vs. $67.8 \%, \mathrm{P}=0.09)$, and regional brain control (38.1\% vs. $12.3 \%, \mathrm{P}=0.049)$ (54).

Several studies have also specifically attempted to address the optimal sequencing of ICIs with SRS. Kiess et al. stratified patients with melanoma brain metastases that underwent SRS with ipilimumab by sequencing: SRS before the first dose of ipilimumab (SRS before; $n=19$ ), SRS between doses of ipilimumab or within 1 month of the last dose of ipilimumab (SRS during; $\mathrm{n}=15$ ), and SRS more than 1 month after the last dose of ipilimumab (SRS after; $\mathrm{n}=12$ ). One-year OS and regional recurrence were $65 \%, 56 \%$, and $40 \%(\mathrm{P}=0.008)$, and $69 \%, 64 \%$, and $92 \%$ $(\mathrm{P}=0.003)$, for the SRS during, SRS before, and SRS after groups, respectively. Interestingly, an increase in brain metastasis diameter to greater than $150 \%$ was seen in half of patients in the SRS during and SRS before groups but only $13 \%$ of the SRS after group (53). Cohen-Inbar et al. grouped patients with melanoma brain metastases that underwent SRS with ipilimumab into two cohorts: SRS before or during ipilimumab (Group A) and SRS after ipilimumab (Group B). Longer local recurrencefree duration was observed in Group A (median 19.6 vs. 
3 months). Interestingly, greater post-SRS perilesional edema was also observed in Group A (49). Skrepnik et al. also looked at timing and sequencing of SRS and ipilimumab in a study of 25 patients with 58 melanoma brain metastases. Patients that underwent SRS between cycles of ipilimumab demonstrated improved time to CNS progression compared to patients that underwent SRS before initiation of ipilimumab or after ipilimumab. Improved time to CNS progression was also observed for patients that underwent SRS within 30 days of an ipilimumab infusion. Similarly, 1-year regional brain control was $83.3 \%$ vs. $37.4 \%$ for patients that underwent SRS within 30 days of ipilimumab compared with greater than 30 days, respectively (63). Finally, in a study of antiPD-1/PD-L1 and SRS in seventeen patients treated to 49 NSCLC brain metastases, 6-month distant brain control was $57 \%$ for patients that underwent SRS before or during anti-PD-1/PD-L1 therapy and $0 \%$ for patients that underwent SRS after anti-PD-1/PD-L1 therapy (67).

Taken together, these preliminary studies suggest a synergy between ICIs and SRS which has been most consistently observed in patients with melanoma brain metastases receiving anti-PD-1 with or without antiCTLA-4 directed therapies. Undergoing SRS before or during ICI therapy might have advantages compared with undergoing SRS after ICI delivery.

\section{ICls and radiosurgery: toxicity}

There have been mixed findings to date regarding whether concurrent ICIs and SRS results in increased rates of toxicity, primarily radiation necrosis. Selected studies are presented in Table 1. In the study by Chen et al. that evaluated the outcomes of 260 patients with brain metastases from melanoma, NSCLC, and RCC, that received SRS alone, SRS with concurrent ICIs (antiCTLA-4, anti-PD-1, or both; given within 2 weeks of SRS), and SRS with non-concurrent ICIs, no difference in toxicity was observed between groups (47). In three studies of SRS with or without ICIs in patients with NSCLC, there were no differences in adverse event rates reported between groups $(61,62,68)$. In the study by Patel et al., 20 patients that received ipilimumab within 4 months of SRS were compared with 34 patients that were treated with SRS alone for melanoma brain metastases and there was no difference in 1-year rates of symptomatic radiation necrosis $(15.0 \% \mathrm{vs}$. $14.7 \%, \mathrm{P}=1.00)(57)$.
On the other hand, several studies have demonstrated an increase in radiation necrosis in the setting of SRS with ICIs $(50,51,69-71)$. Martin et al. identified 480 patients with brain metastases from NSCLC, melanoma, and RCC, that were treated with SRS at a single institution with or without ICIs. There was an increased risk of symptomatic radiation necrosis in patients receiving ICIs (HR 2.56, 95\% CI: 1.35-4.86, $\mathrm{P}=0.004$ ) even after adjustment for primary tumor type, although this association was most pronounced in patients with melanoma (HR 4.02, 95\% CI: 1.17-13.82, $\mathrm{P}=0.03$ ) (69). Similarly, Colaco et al. reviewed the outcomes of 180 patients that underwent SRS for brain metastases from lung ( $n=71)$, melanoma $(n=56)$, breast $(n=27)$, RCC $(n=16)$, colorectal $(n=7)$, and other $(n=3)$, with cytotoxic chemotherapy, targeted therapy, or immunotherapy. Rates of radiation necrosis or tumor-related imaging changes were $37.5 \%$ with immunotherapy, $25.0 \%$ with targeted therapy, and $16.9 \%$ with chemotherapy. Rates of radiation necrosis and tumor-related imaging changes were significantly associated with receipt of immunotherapy [odds ratio (OR) 2.40, 95\% CI: $1.06-$ 5.44, $\mathrm{P}=0.03$ ] (70). Importantly, this study utilized a combined endpoint which included both imaging changes only and symptomatic radiation necrosis. Only 18/39 patients that developed radiation necrosis or treatmentrelated imaging changes had symptomatic radiation necrosis and associations with systemic therapy specific to these patients were not presented (70). Di Perri et al. reviewed the outcomes for 294 patients treated with hypofractionated stereotactic radiotherapy (HFSRT) and found that receipt of immunotherapy within 3 months of HFSRT was associated with development of radiation necrosis (HR 2.69, 95\% CI: 1.10-6.56, P=0.03). Again, in this study only about half of the cases of radiation necrosis were symptomatic (71). Interestingly, the development of radiation necrosis in patients undergoing SRS with ICIs has also been associated with improved OS in some studies $(63,70)$.

In summary, the available data on the risk of radiation necrosis in patients receiving SRS with ICIs are mixed. While several reports show no difference in toxicity $(47,57,61,62,68)$, multiple groups have described an increase in radiation necrosis in this setting $(50,51,69-71)$. Especially in light of the concern for under-reporting of toxicity in retrospective reports, it seems prudent to approach this combination cautiously while we await prospective data. 
Table 1 Select studies with reported toxicity for the combination of stereotactic radiosurgery and immune checkpoint inhibitors

\begin{tabular}{|c|c|c|c|c|c|c|c|c|c|}
\hline Study (ref.) & Site(s) & $\mathrm{ICl}$ target & $\mathrm{N}$ & Arm & \multicolumn{2}{|c|}{$\mathrm{RN}$} & \multicolumn{3}{|c|}{ Other toxicity } \\
\hline \multirow{3}{*}{$\begin{array}{l}\text { Chen et al. } \\
2018,(47)\end{array}$} & \multirow[t]{3}{*}{ Multiple } & \multirow[t]{3}{*}{ PD-1, CTLA-4 } & 28 & SRS + ICI (conc) & \multirow[t]{3}{*}{$3.0 \%$ (path) } & \multirow[t]{3}{*}{ NS } & \multirow{3}{*}{$\begin{array}{c}\text { G3+ acute } \\
\text { CNS }\end{array}$} & $3.00 \%$ & \multirow[t]{3}{*}{ NS } \\
\hline & & & 51 & $\mathrm{SRS}+\mathrm{ICI}$ (non-conc) & & & & $0 \%$ & \\
\hline & & & 181 & SRS without ICI & & & & $4.00 \%$ & \\
\hline $\begin{array}{l}\text { Shepard et al. } \\
2019,(61)\end{array}$ & NSCLC & PD-1 & 34 & $\mathrm{ICl}$-naive & $1 / 34(2.9 \%)$ & - & $\begin{array}{l}\text { Intra-tumoral } \\
\text { hemorrhage }\end{array}$ & 0 & - \\
\hline \multirow{2}{*}{$\begin{array}{l}\text { Singh et al. } \\
2019,(62)\end{array}$} & \multirow[t]{2}{*}{ NSCLC } & \multirow[t]{2}{*}{ PD-1 } & 39 & $\mathrm{SRS}+\mathrm{ICl}$ & $10.20 \%$ & \multirow[t]{2}{*}{0.7} & \multirow[t]{2}{*}{-} & \multirow[t]{2}{*}{-} & \multirow[t]{2}{*}{-} \\
\hline & & & 46 & SRS + chemo & $10.00 \%$ & & & & \\
\hline $\begin{array}{l}\text { Hubbeling } \\
\text { et al. 2018, (68) }\end{array}$ & NSCLC & PD-1, PD-L1 & 35 & $\mathrm{SRS}+\mathrm{ICl}$ & $3.0 \% \mathrm{G} 3 / 4$ & - & $\mathrm{G} 3+\mathrm{AE}$ & $8.00 \%$ & 1.00 \\
\hline $\begin{array}{l}\text { Patel et al. } \\
2017,(57)\end{array}$ & & & 34 & SRS alone & $14.7 \%^{\wedge}$ & & rate at 1 year & $14.70 \%$ & \\
\hline Diao et al. & Mel & CTLA-4 & 51 & $\mathrm{SRS}+\mathrm{ICl}$ & $7.8 \% \mathrm{G} 3 / 4$ & - & - & - & - \\
\hline 2018, (50) & & & 40 & SRS alone & $2.5 \%$ G3/4 & & & & \\
\hline Martin et al. & Multiple & PD-1, CTLA-4 & 115 & $\mathrm{SRS}+\mathrm{ICl}$ & HR $2.56^{\star}$ & 0.004 & - & - & - \\
\hline 2018, (69) & & & 365 & SRS alone & Ref. & & & & \\
\hline Colaco et al. & Multiple & Multiple & 32 & $\mathrm{SRS}+\mathrm{ICl}$ & $37.5 \%^{f}$ & - & - & - & - \\
\hline 2016, (70) & & & 20 & SRS + targeted & $25.0 \%{ }^{f}$ & & & & \\
\hline & & & 83 & SRS + chemo & $16.9 \%{ }^{f}$ & & & & \\
\hline
\end{tabular}

*, HR for symptomatic radiation necrosis; ${ }^{f}$, includes asymptomatic RN/tumor-related imaging changes; $\wedge$, symptomatic radiation necrosis at 1 year. PD-L1, programmed death-ligand 1; CTLA-4, cytotoxic T-lymphocyte associated protein 4; G3+, grade 3 or higher; ICl, immune checkpoint inhibitor; chemo, chemotherapy; Mel, melanoma; NSCLC, non-small cell lung cancer; ref., reference; RN, radiation necrosis; SRS, stereotactic radiosurgery; path, pathologic; HR, hazard ratio; NS, not significant.

\section{Conclusions and future directions}

Overall, studies assessing SRS with concomitant ICIs show promising outcomes in CNS disease control and OS in the retrospective setting. Furthermore, the combination therapy appears overall well tolerated although some groups have observed increased rates of radiation necrosis with this combination and prospective studies are needed to better define the safety profile as well as to better understand the optimal timing and sequencing of this treatment paradigm. Several prospective studies are underway.

\section{Acknowledgments}

Funding: None.

\section{Footnote}

Provenance and Peer Review: This article was commissioned by the editorial office, Translational Cancer Research for the series "Synergy in Action: Novel Approaches to Combining Radiation Therapy and Immunotherapy". The article has undergone external peer review.

Reporting Checklist: The authors have completed the Narrative Review reporting checklist. Available at http:// dx.doi.org/10.21037/tcr-20-3027

Conflicts of Interest: Both authors have completed the ICMJE uniform disclosure form (available at http://dx.doi. org/10.21037/tcr-20-3027). The series "Synergy in Action: 
Novel Approaches to Combining Radiation Therapy and Immunotherapy" was commissioned by the editorial office without any funding or sponsorship. TPR served as the unpaid Guest Editor of the series. The authors have no other conflicts of interest to declare.

Ethical Statement: The authors are accountable for all aspects of the work in ensuring that questions related to the accuracy or integrity of any part of the work are appropriately investigated and resolved.

Open Access Statement: This is an Open Access article distributed in accordance with the Creative Commons Attribution-NonCommercial-NoDerivs 4.0 International License (CC BY-NC-ND 4.0), which permits the noncommercial replication and distribution of the article with the strict proviso that no changes or edits are made and the original work is properly cited (including links to both the formal publication through the relevant DOI and the license). See: https://creativecommons.org/licenses/by-nc-nd/4.0/.

\section{References}

1. Gavrilovic IT, Posner JB. Brain metastases: epidemiology and pathophysiology. J Neurooncol 2005;75:5-14.

2. Stelzer KJ. Epidemiology and prognosis of brain metastases. Surg Neurol Int 2013;4:S192-202.

3. Sperduto PW, Kased N, Roberge D, et al. Summary report on the graded prognostic assessment: an accurate and facile diagnosis-specific tool to estimate survival for patients with brain metastases. J Clin Oncol 2012;30:419-25.

4. Sperduto PW, Mesko S, Li J, et al. Survival in Patients With Brain Metastases: Summary Report on the Updated Diagnosis-Specific Graded Prognostic Assessment and Definition of the Eligibility Quotient. J Clin Oncol 2020;38:3773-84.

5. Non-Small Cell Lung Cancer. In: NCCN Clinical Practice Guidelines in Oncology (NCCN Guidelines). NCCN.org., 2020.

6. Kidney Cancer. In: NCCN Clinical Practice Guidelines in Oncology (NCCN Guidelines). NCCN.org., 2020.

7. Cutaneous Melanoma. In: NCCN Clinical Practice Guidelines in Oncology (NCCN Guidelines). NCCN. org., 2020.

8. Central Nervous System Cancers. In: NCCN Clinical Practice Guidelines in Oncology (NCCN Guidelines). NCCN.org., 2020.

9. Flippot R, Dalban C, Laguerre B, et al. Safety and Efficacy of Nivolumab in Brain Metastases from Renal Cell Carcinoma: Results of the GETUG-AFU 26 NIVOREN Multicenter Phase II Study. J Clin Oncol 2019;37:2008-16.

10. Goldberg SB, Gettinger SN, Mahajan A, et al. Pembrolizumab for patients with melanoma or non-smallcell lung cancer and untreated brain metastases: early analysis of a non-randomised, open-label, phase 2 trial. Lancet Oncol 2016;17:976-83.

11. Goldberg SB, Schalper KA, Gettinger SN, et al. Pembrolizumab for management of patients with NSCLC and brain metastases: long-term results and biomarker analysis from a non-randomised, open-label, phase 2 trial. Lancet Oncol 2020;21:655-63.

12. Kluger HM, Chiang V, Mahajan A, et al. Long-Term Survival of Patients with Melanoma With Active Brain Metastases Treated With Pembrolizumab on a Phase II Trial. J Clin Oncol 2019;37:52-60.

13. Long GV, Atkinson V, Lo S, et al. Combination nivolumab and ipilimumab or nivolumab alone in melanoma brain metastases: a multicentre randomised phase 2 study. Lancet Oncol 2018;19:672-81.

14. Margolin K, Ernstoff MS, Hamid O, et al. Ipilimumab in patients with melanoma and brain metastases: an openlabel, phase 2 trial. Lancet Oncol 2012;13:459-65.

15. Tawbi HA, Forsyth PA, Algazi A, et al. Combined Nivolumab and Ipilimumab in Melanoma Metastatic to the Brain. N Engl J Med 2018;379:722-30.

16. Di Giacomo AM, Ascierto PA, Pilla L, et al. Ipilimumab and fotemustine in patients with advanced melanoma (NIBIT-M1): an open-label, single-arm phase 2 trial. Lancet Oncol 2012;13:879-86.

17. Di Giacomo AM, Ascierto PA, Queirolo P, et al. Threeyear follow-up of advanced melanoma patients who received ipilimumab plus fotemustine in the Italian Network for Tumor Biotherapy (NIBIT)-M1 phase II study. Ann Oncol 2015;26:798-803.

18. Borghaei H, Paz-Ares L, Horn L, et al. Nivolumab versus Docetaxel in Advanced Nonsquamous Non-Small-Cell Lung Cancer. N Engl J Med 2015;373:1627-39.

19. Brahmer J, Reckamp KL, Baas P, et al. Nivolumab versus Docetaxel in Advanced Squamous-Cell Non-Small-Cell Lung Cancer. N Engl J Med 2015;373:123-35.

20. Gandhi L, Rodriguez-Abreu D, Gadgeel S, et al. Pembrolizumab plus Chemotherapy in Metastatic NonSmall-Cell Lung Cancer. N Engl J Med 2018;378:2078-92.

21. Hodi FS, O'Day SJ, McDermott DF, et al. Improved survival with ipilimumab in patients with metastatic melanoma. N Engl J Med 2010;363:711-23. 
22. Motzer RJ, Tannir NM, McDermott DF, et al. Nivolumab plus Ipilimumab versus Sunitinib in Advanced Renal-Cell Carcinoma. N Engl J Med 2018;378:1277-90.

23. Paz-Ares L, Luft A, Vicente D, et al. Pembrolizumab plus Chemotherapy for Squamous Non-Small-Cell Lung Cancer. N Engl J Med 2018;379:2040-51.

24. Reck M, Rodriguez-Abreu D, Robinson AG, et al. Pembrolizumab versus Chemotherapy for PD-L1Positive Non-Small-Cell Lung Cancer. N Engl J Med 2016;375:1823-33.

25. Robert C, Long GV, Brady B, et al. Nivolumab in previously untreated melanoma without BRAF mutation. N Engl J Med 2015;372:320-30.

26. Robert C, Thomas L, Bondarenko I, et al. Ipilimumab plus dacarbazine for previously untreated metastatic melanoma. N Engl J Med 2011;364:2517-26.

27. Socinski MA, Jotte RM, Cappuzzo F, et al. Atezolizumab for First-Line Treatment of Metastatic Nonsquamous NSCLC. N Engl J Med 2018;378:2288-301.

28. Wolchok JD, Chiarion-Sileni V, Gonzalez R, et al. Overall Survival with Combined Nivolumab and Ipilimumab in Advanced Melanoma. N Engl J Med 2017;377:1345-56.

29. Herbst RS, Baas P, Kim DW, et al. Pembrolizumab versus docetaxel for previously treated, PD-L1-positive, advanced non-small-cell lung cancer (KEYNOTE-010): a randomised controlled trial. Lancet 2016;387:1540-50.

30. Gadgeel SM, Lukas RV, Goldschmidt J, et al. Atezolizumab in patients with advanced non-small cell lung cancer and history of asymptomatic, treated brain metastases: exploratory analyses of the phase III OAK study. Lung Cancer 2019;128:105-12.

31. Aoyama H, Shirato H, Tago M, et al. Stereotactic radiosurgery plus whole-brain radiation therapy vs stereotactic radiosurgery alone for treatment of brain metastases: a randomized controlled trial. JAMA 2006;295:2483-91.

32. Brown PD, Ballman KV, Cerhan JH, et al. Postoperative stereotactic radiosurgery compared with whole brain radiotherapy for resected metastatic brain disease (NCCTG N107C/CEC.3): a multicentre, randomised, controlled, phase 3 trial. Lancet Oncol 2017;18:1049-60.

33. Brown PD, Jaeckle K, Ballman KV, et al. Effect of Radiosurgery Alone vs Radiosurgery With Whole Brain Radiation Therapy on Cognitive Function in Patients With 1 to 3 Brain Metastases: A Randomized Clinical Trial. JAMA 2016;316:401-9.

34. Chang EL, Wefel JS, Hess KR, et al. Neurocognition in patients with brain metastases treated with radiosurgery or radiosurgery plus whole-brain irradiation: a randomised controlled trial. Lancet Oncol 2009;10:1037-44.

35. Kocher M, Soffietti R, Abacioglu U, et al. Adjuvant wholebrain radiotherapy versus observation after radiosurgery or surgical resection of one to three cerebral metastases: results of the EORTC 22952-26001 study. J Clin Oncol 2011;29:134-41.

36. Yamamoto M, Serizawa T, Shuto T, et al. Stereotactic radiosurgery for patients with multiple brain metastases (JLGK0901): a multi-institutional prospective observational study. Lancet Oncol 2014;15:387-95.

37. Bernstein MB, Krishnan S, Hodge JW, et al. Immunotherapy and stereotactic ablative radiotherapy (ISABR): a curative approach? Nat Rev Clin Oncol 2016;13:516-24.

38. Ramakrishna R, Formenti S. Radiosurgery and Immunotherapy in the Treatment of Brain Metastases. World Neurosurg 2019;130:615-22.

39. Spiotto M, Fu YX, Weichselbaum RR. The intersection of radiotherapy and immunotherapy: mechanisms and clinical implications. Sci Immunol 2016;1:EAAG1266.

40. Cao Y, Tsien CI, Shen Z, et al. Use of magnetic resonance imaging to assess blood-brain/blood-glioma barrier opening during conformal radiotherapy. J Clin Oncol 2005;23:4127-36.

41. Li YQ, Chen P, Haimovitz-Friedman A, et al. Endothelial apoptosis initiates acute blood-brain barrier disruption after ionizing radiation. Cancer Res 2003;63:5950-6.

42. Nakata H, Yoshimine T, Murasawa A, et al. Early bloodbrain barrier disruption after high-dose single-fraction irradiation in rats. Acta Neurochir (Wien) 1995;136:82-6; discussion 86-7.

43. Acharya S, Mahmood M, Mullen D, et al. Distant intracranial failure in melanoma brain metastases treated with stereotactic radiosurgery in the era of immunotherapy and targeted agents. Adv Radiat Oncol 2017;2:572-80.

44. Ahmed KA, Abuodeh YA, Echevarria MI, et al. Clinical outcomes of melanoma brain metastases treated with stereotactic radiosurgery and anti-PD-1 therapy, antiCTLA-4 therapy, BRAF/MEK inhibitors, BRAF inhibitor, or conventional chemotherapy. Ann Oncol 2016;27:2288-94.

45. An Y, Jiang W, Kim BY, et al. Stereotactic radiosurgery of early melanoma brain metastases after initiation of anti-CTLA-4 treatment is associated with improved intracranial control. Radiother Oncol 2017;125:80-8.

46. Anderson ES, Postow MA, Wolchok JD, et al. Melanoma brain metastases treated with stereotactic radiosurgery and concurrent pembrolizumab display marked regression; 
efficacy and safety of combined treatment. J Immunother Cancer 2017;5:76.

47. Chen L, Douglass J, Kleinberg L, et al. Concurrent Immune Checkpoint Inhibitors and Stereotactic Radiosurgery for Brain Metastases in Non-Small Cell Lung Cancer, Melanoma, and Renal Cell Carcinoma. Int J Radiat Oncol Biol Phys 2018;100:916-25.

48. Choong ES, Lo S, Drummond M, et al. Survival of patients with melanoma brain metastasis treated with stereotactic radiosurgery and active systemic drug therapies. Eur J Cancer 2017;75:169-78.

49. Cohen-Inbar O, Shih HH, Xu Z, et al. The effect of timing of stereotactic radiosurgery treatment of melanoma brain metastases treated with ipilimumab. J Neurosurg 2017;127:1007-14.

50. Diao K, Bian SX, Routman DM, et al. Stereotactic radiosurgery and ipilimumab for patients with melanoma brain metastases: clinical outcomes and toxicity. J Neurooncol 2018;139:421-9.

51. Diao K, Bian SX, Routman DM, et al. Combination ipilimumab and radiosurgery for brain metastases: tumor, edema, and adverse radiation effects. J Neurosurg 2018;129:1397-406.

52. Gatterbauer B, Hirschmann D, Eberherr N, et al. Toxicity and efficacy of Gamma Knife radiosurgery for brain metastases in melanoma patients treated with immunotherapy or targeted therapy-A retrospective cohort study. Cancer Med 2020;9:4026-36.

53. Kiess AP, Wolchok JD, Barker CA, et al. Stereotactic radiosurgery for melanoma brain metastases in patients receiving ipilimumab: safety profile and efficacy of combined treatment. Int J Radiat Oncol Biol Phys 2015;92:368-75.

54. Lehrer EJ, Peterson J, Brown PD, et al. Treatment of brain metastases with stereotactic radiosurgery and immune checkpoint inhibitors: An international metaanalysis of individual patient data. Radiother Oncol 2019;130:104-12.

55. Minniti G, Anzellini D, Reverberi C, et al. Stereotactic radiosurgery combined with nivolumab or Ipilimumab for patients with melanoma brain metastases: evaluation of brain control and toxicity. J Immunother Cancer 2019;7:102.

56. Nguyen SM, Castrellon A, Vaidis O, et al. Stereotactic Radiosurgery and Ipilimumab Versus Stereotactic Radiosurgery Alone in Melanoma Brain Metastases. Cureus 2017;9:e1511.

57. Patel KR, Shoukat S, Oliver DE, et al. Ipilimumab and stereotactic radiosurgery versus stereotactic radiosurgery alone for newly diagnosed melanoma brain metastases. Am J Clin Oncol 2017;40:444-50.

58. Qian JM, Yu JB, Kluger HM, et al. Timing and type of immune checkpoint therapy affect the early radiographic response of melanoma brain metastases to stereotactic radiosurgery. Cancer 2016;122:3051-8.

59. Robin TP, Breeze RE, Smith DE, et al. Immune checkpoint inhibitors and radiosurgery for newly diagnosed melanoma brain metastases. J Neurooncol 2018;140:55-62.

60. Schapira E, Hubbeling H, Yeap BY, et al. Improved overall survival and locoregional disease control with concurrent PD-1 pathway inhibitors and stereotactic radiosurgery for lung cancer patients with brain metastases. Int J Radiat Oncol Biol Phys 2018;101:624-9.

61. Shepard MJ, Xu Z, Donahue J, et al. Stereotactic radiosurgery with and without checkpoint inhibition for patients with metastatic non-small cell lung cancer to the brain: a matched cohort study. J Neurosurg 2019. [Epub ahead of print]. doi: 10.3171/2019.4.JNS19822.

62. Singh C, Qian JM, James BY, et al. Local tumor response and survival outcomes after combined stereotactic radiosurgery and immunotherapy in non-small cell lung cancer with brain metastases. J Neurosurg 2019;132:512-7.

63. Skrepnik T, Sundararajan S, Cui H, et al. Improved time to disease progression in the brain in patients with melanoma brain metastases treated with concurrent delivery of radiosurgery and ipilimumab. Oncoimmunology 2017;6:e1283461.

64. Yusuf MB, Amsbaugh MJ, Burton E, et al. Peri-SRS administration of immune checkpoint therapy for melanoma metastatic to the brain: investigating efficacy and the effects of relative treatment timing on lesion response. World Neurosurgery 2017;100:632-640.e4.

65. Knisely JP, James BY, Flanigan J, et al. Radiosurgery for melanoma brain metastases in the ipilimumab era and the possibility of longer survival. J Neurosurg 2012;117:227-33.

66. Silk AW, Bassetti MF, West BT, et al. Ipilimumab and radiation therapy for melanoma brain metastases. Cancer Med 2013;2:899-906.

67. Ahmed KA, Kim S, Arrington J, et al. Outcomes targeting the PD-1/PD-L1 axis in conjunction with stereotactic radiation for patients with non-small cell lung cancer brain metastases. J Neurooncol 2017;133:331-8.

68. Hubbeling HG, Schapira EF, Horick NK, et al. Safety of combined PD-1 pathway inhibition and intracranial radiation therapy in non-small cell lung cancer. J Thorac 
Oncol 2018;13:550-8.

69. Martin AM, Cagney DN, Catalano PJ, et al. Immunotherapy and symptomatic radiation necrosis in patients with brain metastases treated with stereotactic radiation. JAMA Oncol 2018;4:1123-4.

70. Colaco RJ, Martin P, Kluger HM, et al. Does immunotherapy increase the rate of radiation necrosis after radiosurgical treatment of brain metastases? J Neurosurg 2016;125:17-23.

71. Di Perri D, Tanguy R, Malet C, et al. Risk of radiation necrosis after hypofractionated stereotactic radiotherapy (HFSRT) for brain metastases: a single center retrospective study. J Neurooncol 2020;149:447-53.
Cite this article as: Moskalenko M, Robin TP. Narrative review of immune checkpoint inhibitors and radiation therapy for brain metastases. Transl Cancer Res 2021;10(5):2527-2536. doi: $10.21037 /$ tcr-20-3027 